\title{
A Novel Texture based Skin Melanoma Detection using Color GLCM and CS-LBP Feature
}

\author{
Rohini S. Mahagaonkar \\ Dept. of Comp. Sc. \& Eng \\ P.D.A College of Engineering \\ Gulbarga, Karnataka, India
}

\author{
Shridevi Soma, PhD \\ Dept. of Comp. Sc. \& Eng \\ P.D.A College of Engineering \\ Gulbarga, Karnataka, India
}

\begin{abstract}
Microscopic analysis of skin cancer images for detection of melanoma or lesion has drawn significant medical interest over last few years. Various kinds of skin abnormalities are not well detectable from mear observation of the microscopic images. Most of the detection of skin abnormality has been relied on the variation of the texture of a specific region of the skin in comparison to the neighborhood of the area. Therefore image processing has been widely used for such detection techniques. However such detection technique fails to distinguish between different types of abnormalities. In case of a blister in the skin which appears the same abnormal way that a probable tumor or a sun burn in a specific area in the skin. Due to variation of the skin texture in the presence of any common abnormality like sun burn, blister, etc, it is extremely difficult for the present systems to differentiate a melanoma or a skin tumor or a cancer from the other skin diseases. Therefore in this work a unique system is developed to detect skin abnormality using a machine learning framework to classify a skin abnormality as melanoma more effectively. The proposed machine learning system relies on extraction of advanced texture features such as harlick GLCM features and CS LBP features in order to detect melanoma in the dermoscopy skin images. A total of 300 images from standard dataset dermquest.com are considered to carry out experimentation, and accuracy of the system using $\mathrm{KNN}$ is $79.7315 \%$ and SVM is $84.7615 \%$.
\end{abstract}

\section{Keywords}

Texture pattern, GLCM, CS LBP, KNN, SVM

\section{INTRODUCTION}

Malignant melanoma is a type of cancer that in almost all cases starts in pigment cells (melanocytes) in the skin. Most of the persons when they think of melanoma think of a dark raised lesion, after all these tumors grow from the pigmentation of the cells. But some melanomas lose pigment, lacking the dark pigment partially or completely, and appear pink, white or tan. Melanoma is considered to be the deadliest of skin cancers. According to the American Cancer Society (ACS), about 76,380 (46,870 men and 29,510 women) new cases of melanomas are estimated to be diagnosed in the year 2016. The incidence of melanoma has been rising every year. Many of these lives could be saved if melanoma were to be detected at the earliest stage, when it is easily curable. A number of studies using various technologies are being conducted around the world for the early detection of melanoma. Dermoscopy is a method of acquiring a magnified and illuminated image of a region of skin for increased clarity of the spots on the skin. The imaging instrument used for this purpose is called a dermatoscope. Dermatoscopes are of two types: contact, using a layer of gel/oil applied between skin and dermatoscope, and non-contact, with no skin contact and no fluid. Non-contact images and some contact images use cross-polarized light from the dermatoscope to acquire the image. Because of their illumination and magnification, dermoscopy images are widely used in the analysis and examination of skin lesions. Segmentation of the lesion means separating that region (lesion) from the normal skin region (non-lesion). Lesion segmentation is a very important step in the analysis of dermoscopy images for it allows the identification of various global morphological features specific to the lesion and at the same time provides a confined region for segmentation of various local clinical features at a later stage.

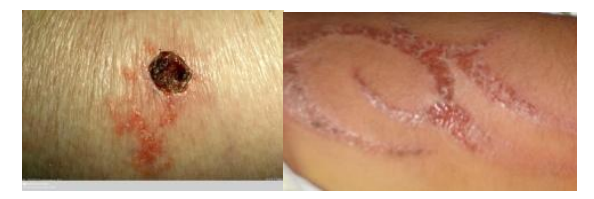

Fig 1: Sample of abnormal skin images

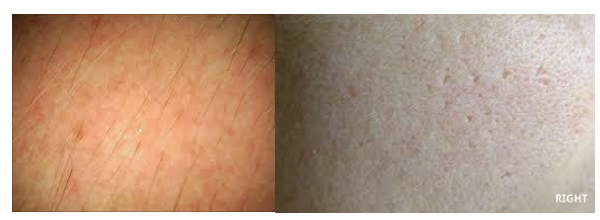

Fig 2: Sample of normal skin images

\section{RELATED WORK}

Previous survey $[3,5,6,8,9,14,15,16,17,18,20]$ were based on the early detection of the melanoma using different methods and techniques and $[1,7,19]$ were based on the classification of the lesions and $[2,4,10,11,12,13]$ were based on the color identification in the melanocytic lesions and also segmentation of the particular areas and computer aided system for the malignant melanomas. [3] Omid Sarrafzade et. al.have proposed skin lesion detection using wavelet transform. Morphology operations are used for the segmentation of the image and the results show that it has the low percentage of the border error [5] Tarun Wadhawan et. Al has proposed the detection of the blue-whitish veil using color descriptors. It is combined with the regression feature that can provide the sensitivity and specificity of $95.64 \%$ and $72.30 \%$ respectively. [6] Mariam A.Sheha et. al have proposed automatic melanoma detection using texture analysis. The features extracted are the gray-level co-occurrence matrix using the multilayer perception classifier. Two techniques have been used i.e. automatic MLP and traditional MLP.The obtained accuracy was 92\%. [1] Hitoshi Iyatomi et al. have proposed classification of the two different lesions. The two features the skewness of bright region in the tumor and the difference between the average intensity in the peripheral part of the tumor were efficient for the classification. The 
detection accuracy obtained is $98 \%$ and $86.6 \%$ respectively. [7] Jorge S. Marques et. al. have proposed texture and the color classification system. Histograms wing descriptors. The result obtained by combining the features was $94.1 \%$ and 77.4\% respectively.[19] Reda kasmi et al. have proposed classification of the lesions using the ABCD rule. Asymmetry, border, color and Dermoscopic structure are the best descriptors for the automatic classification of the lesions. The accuracy obtained was $92.8 \%$ and $90.3 \%$ respectively. [11] J. Jaworek-Korjakowska et al. have proposed determination of the border irregularity in the dermoscopy color images. Their system includes the image enhancement, segmentation, border detection and classification. The accuracy obtained was $79 \%$ for 300 dataset. [13] S. Sabbaghi et. al have proposed automated color identification in lesions. Technique QuadTree decomposition was used for the automatic identification. The accuracy obtained using $\mathrm{NIH}$ and $\mathrm{PH} 2$ dataset was $93 \%$.

\section{PROPOSED WORK}

The proposed methodology of the detection of the skin image as normal or abnormal is shown in the Figure 3.The proposed system includes various steps of image processing such as Pre-processing, Segmentation,featureextraction,classification.

The work is been implemented using Matlab 2016.

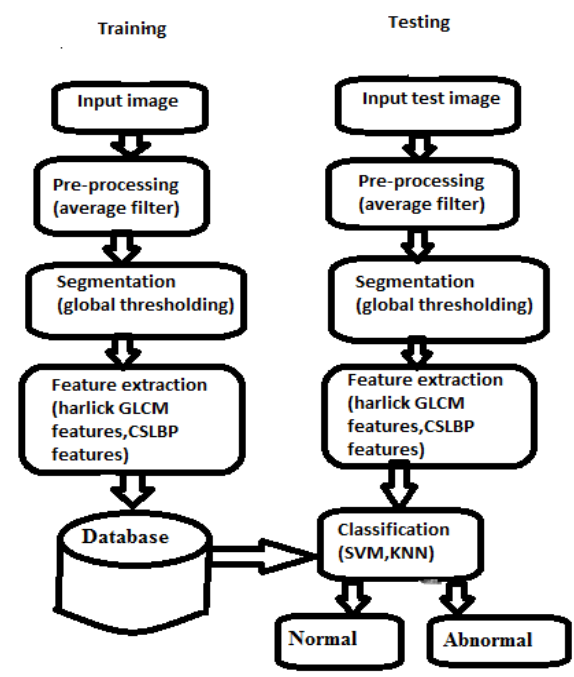

Fig 3: Block diagram of the proposed system

\subsection{Database Collection}

For the study set the dermoscopy images were been collected from standard data set. The standard dataset has been collected from the dermquest.com. Regarding all images are been divided into two classes $25 \%$ for training set and $75 \%$ for test set.

\subsection{Pre-processing}

This study based on the texture pattern the preprocessing technique is been applied to the images that is the average filter. Hence it is a simple process because it removes the pores and the other texture from the skin image also the image is been converted into HSV because in RGB image the color variations are significantly high but in the HSV image the color variation decreases significantly.

\subsection{Segmentation}

In segmentation thresholding based segmentation technique is been used. The threshold based technique used is the global thresholding. Global thresholding is setting an intensity value (threshold) such that all pixels having intensity value below the threshold belong to one phase, the remaining belong to the other. The image is been segmented into 2 blocks with probable tumor and non-probable tumor area. It means dividing the image into two parts one which matches the condition and one which doesn't match the condition.

\subsection{Feature Extraction}

All the features extracted here are texture features the two main features extracted here are Harlick GLCM features and CSLBP features.

3.4.1 Harlick GLCM features: The harlick Gray level co-occurrence matrix (GLCM) is a powerful tool for the image feature extraction by mapping the grey level cooccurrence probabilities based on the spatial relations of pixels in different angular directions. The features extracted based on GLCM are: angular second moment (energy), contrast, correlation, variance, inverse difference moment (homogeneity), sum average, sum variance, sum entropy, entropy, difference variance, difference entropy, information measure of correlation, maximal correlation coefficient.

Table 1 gives the formula to calculate each of the Harlick GLCM features.

Table 1: GLCM features

\begin{tabular}{|c|c|}
\hline Angular Second Moment & $\sum_{i} \sum_{j} p(i, j)^{2}$ \\
\hline Contrast & $\sum_{n=0}^{N_{g}-1} n^{2}\left\{\sum_{i=1}^{N_{g}} \sum_{j=1}^{N_{g}} p(i, j)\right\},|i-j|=n$ \\
\hline Correlation & $\begin{array}{r}\frac{\sum_{i} \sum_{j}(i j) p(i, j)-\mu_{x} \mu_{y}}{\sigma_{x} \sigma_{y}} \\
\text { where } \mu_{x}, \mu_{y}, \sigma_{x}, \text { and } \sigma_{y} \\
\text { are the means and std. deviations } \\
\text { of } p_{x} \text { and } p_{y}, \text { the partial probability } \\
\text { density functions }\end{array}$ \\
\hline Sum of Squares: Variance & $\sum_{i} \sum_{j}(i-\mu)^{2} p(i, j)$ \\
\hline Inverse Difference Moment & $\sum_{i} \sum_{j} \frac{1}{1+(i-j)^{2}} p(i, j)$ \\
\hline Sum Average & $\begin{array}{l}\sum_{i=2}^{2 N_{g}} i p_{x+y}(i) \\
\text { where } x \text { and } y \text { are the coordinates (row and } \\
\text { column) of an entry in the co-occurrence matrix, } \\
\text { and } p_{x+y}(i) \text { is the probability of co-occurrence } \\
\text { matrix coordinates summing to } x+y\end{array}$ \\
\hline Sum Variance & $\sum_{i=2}^{2 N_{g}}\left(i-f_{8}\right)^{2} p_{x+y}(i)$ \\
\hline Sum Entropy & $-\sum_{i=2}^{2 N_{g}} p_{x+y}(i) \log \left\{p_{x+y}(i)\right\}=f_{8}$ \\
\hline Entropy & $-\sum_{i} \sum_{j} p(i, j) \log (p(i, j))$ \\
\hline Difference Variance & $\sum_{i=0}^{N_{g}-1} i^{2} p_{x-y}(i)$ \\
\hline Difference Entropy & $-\sum_{i=0}^{N_{g}-1} p_{x-y}(i) \log \left\{p_{x-y}(i)\right\}$ \\
\hline Info. Measure of Correlation 1 & $\frac{H X Y-H X Y 1}{\max \{H X, H Y\}}$ \\
\hline Info. Measure of Correlation 2 & $\begin{array}{r}(1-\exp [-2(H X Y 2-H X Y)])^{\frac{1}{2}} \\
\text { where } H X Y=-\sum_{i} \sum_{j} p(i, j) \log (p(i, j)), H X, \\
H Y \text { are the entropies of } p_{x} \text { and } p_{y}, H X Y 1= \\
-\sum_{i} \sum_{j} p(i, j) \log \left\{p_{x}(i) p_{y}(j)\right\} H X Y 2= \\
-\sum_{i} \sum_{j} p_{x}(i) p_{y}(j) \log \left\{p_{x}(i) p_{y}(j)\right\}\end{array}$ \\
\hline Max. Correlation Coeff. & $\begin{array}{l}\text { Square root of the second largest eigenvalue of } \mathbf{Q} \\
\text { where } \mathbf{Q}(i, j)=\sum_{k} \frac{p(i, k) p(j, k)}{p_{x}(i) p_{y}(k)}\end{array}$ \\
\hline
\end{tabular}

\subsubsection{LBP features: LBP is a visual descriptor used for} classification. It is a powerful feature for the texture analysis.

The procedure for extracting local binary pattern (LBP) features is as follows.

1) Divide the window into cells.

2) For each pixel in a cell, compare the pixel to each of its neighbor's, also the pixels along a circle, i.e. clockwise or counter-clockwise. 
3) Where the center pixel's value is greater than the neighbor's value, write " $0 "$. Otherwise, write " $1 "$.

4) Compute the histogram of each pixel of various frequencies.

5) Normalize the histogram. (Optional)

6) Concatenate (normalized) histograms of all cells. Hence the feature vector of the entire window is been obtained.

CSLBP (circular shift local binary pattern): In the CSLBP first convert pixel into 30, 60, 90,120 and 180 degree .So from 0 to 360 degree, rotate the pixels and calculate the LBP of the each degree then take the average.

\subsection{Classification}

\section{Algorithm}

Algorithm 1 gives the flow of Testing, Training and Classification procedure used in the proposed work.

\section{Algorithm 1: Identification of skin Image}

Input: Different types of skin images

Output: Identification of the skin image as normal and abnormal

\section{Method: Harlick GLCM and CSLBP features}

Feature vector of size: 14 Harlick features and 16 CSLBP features.

\section{Train Phase:}

Start

Step 1: Input the skin image.

Step 2: Perform preprocessing of image using average filter.

Step 3: Perform global thresholding based Segmentation for the primary detection.

Step 4: Extract Harlick GLCM and CSLBP features of segmented image.

Step 5: Store all the features as a feature vector in train library

End

\section{Test Phase:}

Start

Step 1: Input the skin image.

Step 2: Perform preprocessing of image using average filter.

Step 3: Perform global thresholding based segmentation for the primary detection.

Step 4: Extract Harlick GLCM and CSLBP features of Segmented Image.

Step 5: Compare the test image features with the database features.

Step 6: Identify the input test image using SVM and KNN Classifier as normal or abnormal.

End

\section{RESULT AND DISCUSSION}

The results of classification on images that acquired by the dermoscopy technique. GLCM and CSLBP features were used for feature extraction.14 harlick GLCM features and 16 CSLBP features were considered. To train and test the system the KNN and SVM classifiers were used to classify the image as normal and abnormal. The proposed method trained with $25 \%$ and tested with $75 \%$ of the total number of images.

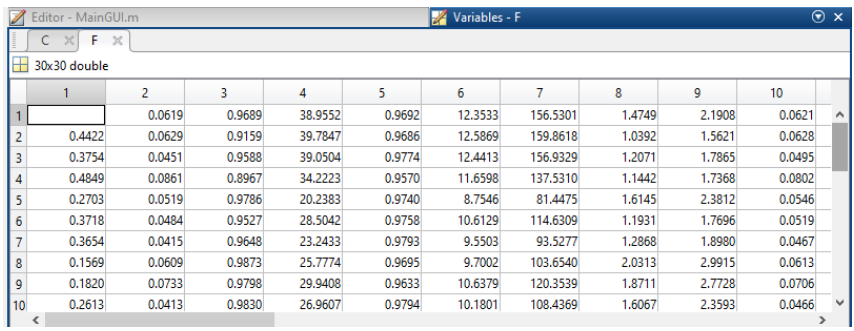

Fig 4: Sample of harlick GLCM and CSLBP features

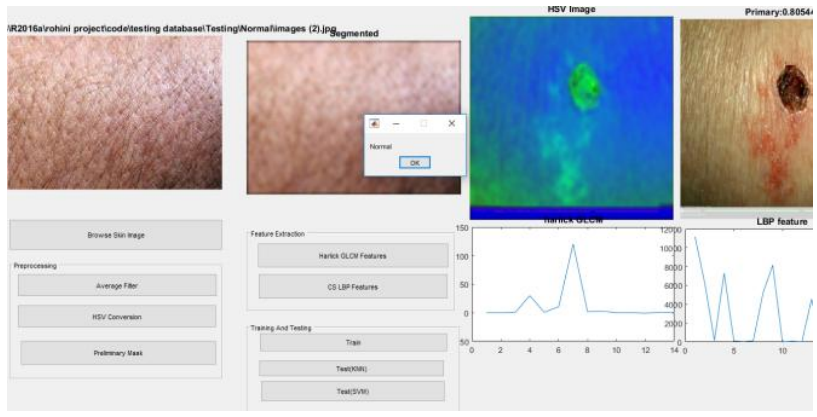

Fig 5: Test result for the normal image

Table 2: Performance of SVM classifier

\begin{tabular}{|c|c|c|c|c|c|}
\hline Skin Classes & train & test & Correctly identified & incorrectly identified & ccuracy(\%) \\
\hline Normal & 30 & 70 & 58 & 12 & 82.85 \\
\hline Abnormal & 50 & 150 & 130 & 20 & 86.66 \\
\hline
\end{tabular}

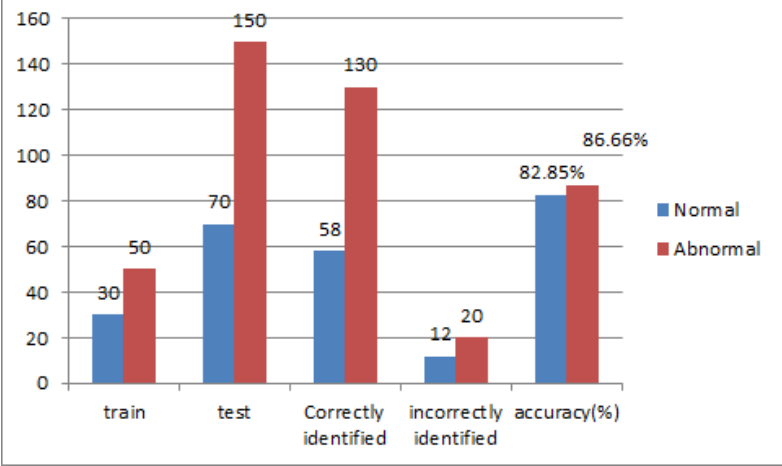

Fig 6: Performance using SVM classifier

Table 2 and 3 gives the performance details of SVM and KNN classifier for the data set considered for experimentation.

Table 3: Performance of KNN classifier

\begin{tabular}{|c|c|c|c|c|c|}
\hline Skin Classes & train & test & Correctly identified & incorrectly identified & accuracyl\% \\
\hline Normal & 30 & 70 & 53 & 17 & 76.13 \\
\hline Abnormal & 50 & 150 & 125 & 25 & 83.33 \\
\hline
\end{tabular}




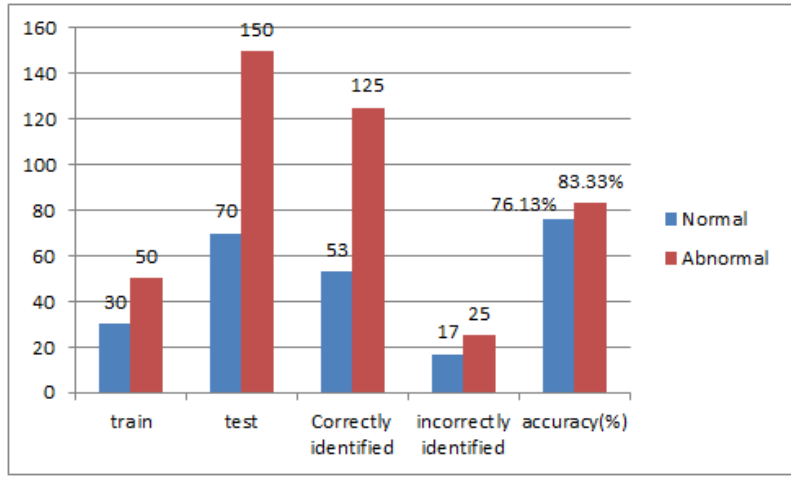

Fig 7: Performance of KNN classifier

\begin{tabular}{|c|c|c|c|}
\hline Type of classifier & Accuracy of Normal Skin Images(\%) & Accuracy of Abnormal Skin Images(\%) & peral accuracy/\% \\
\hline SVM & 82.85 & 86.66 & 84.76 \\
\hline KNN & 76.13 & 83.33 & 79.73 \\
\hline
\end{tabular}

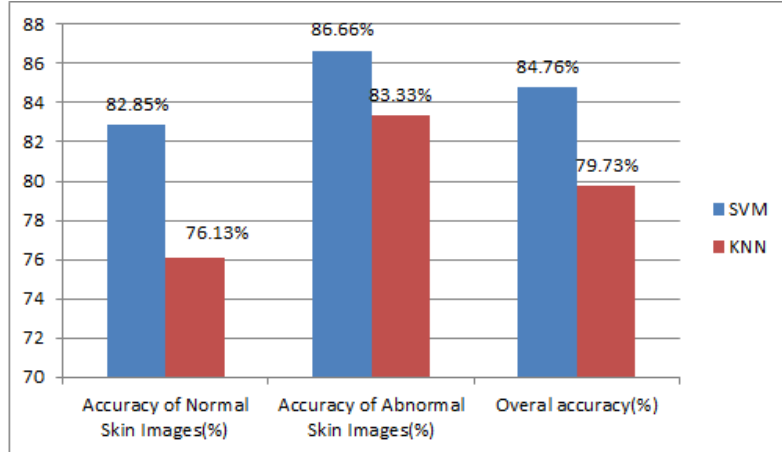

Fig 8: Overall accuracy of SVM and KNN classifiers

\section{CONCLUSION AND FUTURE SCOPE}

With the advancement in the computer vision technology and the cloud based record maintaince, machine learning technique for automated diagnosis of the medical images is gaining significant popularity. Various machine learning based skin cancer detection techniques have been proposed in the past. Most of the techniques were dependent on threshold based. The problem with the threshold based technique is that they are more susaptable to the variation in the skin color and texture due to change in gender, age, and ethnicity and so on. Therefore in proposed work instead of adaptive threshold based technique for the skin cancer detection a system is developed which machine learning based skin cancer detection combines the best practices of the segmentation by pre-processing the image with segmentation step followed by a texture based representation of the image. As texture is considered to be one of the better descriptors of the skin cancer as well as any abnormality in the skin area the proposed system is found to be extremely accurate by considering Harlick GLCM \& CSLBP features. The system accuracy of the proposed system is found to be $79.7315 \%$ for KNN classifier and $84.7615 \%$ for SVM classifier. From this it can conclude that SVM classifier performs better for skin cancer detection. Future scope of this work is to incorporate cancer stage identification like benign and malignant. Such kind of multi-level classification can be performed by adapting multi-level classifier.

\section{REFERENCES}

[1] Hitoshi Iyatomi, Kerri-Ann Norton, M.Emre Celebi Gerald Schaefer, Masaru Tanaka, and Koichi Ogawa "Classification of melanocytic skin lesions from NonMelanocytic lesions". IEEE 2010

[2] Stein Olav Skrøvseth Thomas R. Schopf, Kevin Thon,Maciel Zortea, Marc Geilhufe, Kajsa Møllersen, Herbert M. Kirchesch and Fred Godtliebsen "A computer aided diagnostic system for malignant melanomas" IEEE 2010 .

[3] Omid Sarrafzade Mohammad Hossein Miran Baygi Pejhman Ghassemi "Skin Lesion Detection in Dermoscopy Images Using Wavelet Transform and Morphology Operations" 17th Iranian Conference November 2010 .

[4] Gianluca Sforza, Giovanna CastellanoR. Joe Stanley William V. Stoecker, Jason Hagerty "Adaptive segmentation of gray areas in dermoscopy images" 2011 IEEE 2011.

[5] Tarun Wadhawan, Rui Hu, and George Zouridakis, "Detection of Blue-Whitish Veil in Melanoma using Color Descriptors" 978-1-4577-2177-9/12/\$25 (C) 2012 IEEE.

[6] Mariam A.Sheha Mai S.Mabrouk Amr Sharawy "Automatic Detection of Melanoma Skin Cancer using Texture Analysis" International Journal of Computer Applications (0975 - 8887) Volume 42- No.20, March 2012

[7] Jorge S. Marques1 Catarina Barata1 Teresa Mendonc sa2 "On the Role of Texture and Color in the Classification of Dermoscopy Images" 34th Annual International Conference 2012 IEEE.

[8] Maryam Sadeghi, Tim K. Lee David McLean, Harvey Lui, and M. Stella Atkins "Detection and Analysis of Irregular Streaks in Dermoscopic Images of Skin Lesions" IEEE TRANSACTIONS ON MEDICAL IMAGING, VOL.32, NO.5, MAY2013

[9] Catarina Barata, Margarida Ruela, Mariana Francisco, Teresa Mendonça, and Jorge S. Marques "Two Systems for the Detection of Melanomas in Dermoscopy Images Using Texture and Color Features" 2013 IEEE.

[10] Di Ming, Quan Wen, Juan Chen Wenhao liu "A Generalized Fusion Approach for Segmenting Dermoscopy Images Using Markov Random Field" 9781-4799-2764-7/13/\$31.00 @2013 IEEE.

[11] J. Jaworek-Korjakowska and R. Tadeusiewicz "Determination of Border Irregularity in Dermoscopic Color Images of Pigmented Skin Lesions" 2014 IEEE.

[12] Catarina Barata1 Mario A. T. Figueiredo2 M. Emre Celebi3 Jorge S. Marques1 "COLOR IDENTIFICATION IN DERMOSCOPY IMAGES USING GAUSSIAN MIXTURE MODELS" 978-14799-2893-4/14/\$31.00 @2014 IEEE.

[13] S. Sabbaghi, M. Aldeen, R. Garnavi, G. Varigos, C. Doliantis and J. Nicolopoulos "Automated Colour Identification in Melanocytic Lesions" 978-1-4244-92701/15/\$31.00 () 2015 IEEE. 
[14] Omar Abuzaghleh, Miad Faezipour and Buket D. Barkana "A Comparison of Feature Sets for an Automated Skin Lesion Analysis System for Melanoma Early Detection and Prevention” 2015 IEEE.

[15] Priyadarshini D1, Rengini D 2 "Automatic Melanoma Detection Using Local Binary Pattern and Support Vector Machine" DOI: 10.15680/IJIRCCE.2015. 0309086.

[16] Saudamini S. Jivtode1, Amit Ukalkar2 "Neural Network Based Detection of Melanoma Skin Cancer" Paper ID: NOV163985 Volume 5 Issue 6, June 2016.

[17] PeymanSabouri and Hamid Gholam Hosseini "Lesion Border Detection Using Deep Learning" 978-1-50900623-6/16/\$31.00 c 2016 IEEE.
[18] Lei Bi1, Jinman Kim1, Euijoon Ahn1, Dagan Feng and Michael Fulham "Automatic Melanoma Detection via Multi-scale Lesion-biased Representation and Joint Reverse Classification" 2016 IEEE.

[19] Reda Kasmi, Karim Mokrani "Classification of malignant melanoma and benign skin lesions" IET Image Process., 2016, Vol. 10, Iss. 6, pp. 448-455.

[20] Suganya R “An Automated Computer Aided Diagnosis of Skin Lesions Detection and Classification for DermoscopyImages" 2016 Fifth International Conference 978-1-4673-9802-2/16/\$31.00@ 2016 IEEE. 